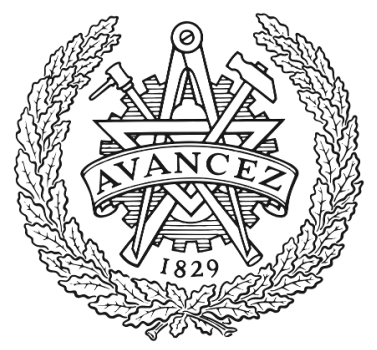

CHALMERS

UNIVERSITY OF TECHNOLOGY

\title{
Key Drivers and Future Scenarios of Local Energy and Flexibility Markets
}

Downloaded from: https://research.chalmers.se, 2023-04-26 08:15 UTC

Citation for the original published paper (version of record):

Mirzaei Alavijeh, N., Fotouhi, A., Steen, D. et al (2021). Key Drivers and Future Scenarios of Local Energy and Flexibility Markets. 2021 IEEE Madrid PowerTech, PowerTech 2021 - Conference Proceedings. http://dx.doi.org/10.1109/PowerTech46648.2021.9494828

N.B. When citing this work, cite the original published paper. 


\title{
Key Drivers and Future Scenarios of Local Energy and Flexibility Markets
}

\author{
Nima Mirzaei Alavijeh, Mohammad Ali Fotouhi Ghazvini, David Steen, Le Anh Tuan, Ola Carlson \\ Division of Electrical Power Engineering \\ Department of Electrical Engineering \\ Chalmers University of Technology \\ Gothenburg, Sweden \\ Email: \{nima.mirzaei, ali.fotouhi, david.steen, tuan.le, ola.carlson\}@chalmers.se
}

\begin{abstract}
This paper explores the key drivers of local energy and flexibility markets, develops a set of plausible future scenarios for these markets, and analyzes the scenarios' impact. The results can provide insight to policymakers, researchers, system operators, and aggregators in a better design and more successful implementation of local markets. This study is based on the well-established scenario planning technique of "intuitive logics" and it is conducted by means of qualitative methods, surveys, and cross-impact analysis. Results explore and rank the impact and uncertainty of 20 key factors and trends which can affect the future of local energy and flexibility markets. The results show that factors related to the availability of active and smart end-users, and regulatory incentives are the most impactful and uncertain ones in the future of local markets. Four future scenarios are introduced based on these factors and their impacts are discussed.
\end{abstract}

Index Terms-Local flexibility markets, local energy markets, scenario planning, distribution system operator, service market, aggregator, flexibility

\section{INTRODUCTION}

The operational challenges in distribution networks are arising due to increasing penetration of intermittent energy resources [1], electrification of the transport and heating sectors, and the transformation of the networks from uni-directional centralized systems to bi-directional decentralized systems [2]. These challenges are, among others, balancing challenges, congestions, voltage stability issues, and new peaks. The concept of local markets for trading energy and flexibility is suggested as a potential solution to overcome these challenges. A market can be defined as a platform that gathers different potential buyers, sellers, and retailers of a specific product [3]. In the context of local energy and flexibility markets, the product is energy or flexibility, which is traded in a geographically limited area. Flexibility is the possibility of adjusting patterns of generation and consumption in response to a signal to provide different grid services. It is technically defined as a power modification activated at a defined time for a specified duration at a specific location [2]. Flexibility can be deployed by the system operator to maintain system balance while satisfying the grid capacity constraints and guaranteeing the safe grid operation [4]. The geographically limited areas

The work leading to this paper is from work-package 2 of FLEXIGRID project that is funded by the European Community's Horizon 2020 Framework Programme under grant agreement no. 864048. in local energy and flexibility markets can be neighborhoods, small cities, towns, or communities [5]. Having such markets at a local level can facilitate enabling small and distributed flexibility, managing congestions at the distribution level, and increasing awareness and engagement among end-users [6]. Further information about the state of the art for the local markets can be found, among others, in [1], [2], [7], [8].

As the research is ongoing on the development and evaluation of local markets, understanding the key factors and trends that impact the future of local markets can contribute to a better design and a successful implementation of such markets. Scenario planning methods can be used to explore the key factors and trends and provide insight to different stakeholders, such as policymakers, system operators, service providers, and researchers. Scenarios are the possible forms of the future that provide narratives for a context and facilitate decision-making [9]. However, it is important to keep in mind that scenarios are not predictions of the future, but rather an exploration of the drivers of change and multiple plausible future situations [9], [10]. Scenario planning provides a structured conversation to familiarize decision-makers with different uncertainties and to build a shared understanding of such uncertainties [11].

This paper aims to explore the key drivers of local energy and flexibility markets, to develop a set of plausible future scenarios for the local markets, and to analyze the impact of these scenarios. It provides insights for policymakers, researchers, and other stakeholders to contribute to a better design and a more successful implementation of local markets, using well-established scenario planning methods.

Scenario planning methods are used in different research areas. In the energy systems area, Chen et al. [12] have integrated the Delphi method within scenario planning methods to identify the key drivers and factors in China's renewable energy development and develop scenarios for renewable energy development on a time-horizon of 2030. Kuiper et al. [13] developed and analyzed scenarios for the future of a Dutch distribution system operator (DSO) for the year 2030 using an 8-step scenario development approach via the intuitive logic method. Alizadeh et al. [14] proposed an integrated scenariobased planning method including various methods such as Delphi, political, economic, social, and technological analysis, and cross-impact analysis. Iran's energy industry is used as a 
test-case for the method. Schuckmann et al. [15] identifies and assesses various factors that affect the future of the transport sector, and develops four different probable future scenarios while discussing managerial, strategy and policy implications. Shiftan et al. [16] have also used the scenario approach to explore the future developments of Tel-Aviv and extracting the key elements in forming the future of its transportation.

Scenario planning methods have not been used broadly in the research area of energy management systems for familiarizing different stakeholders with the uncertainties in the implementation of new concepts. Moreover, there are no studies about local energy and flexibility markets that use such methods to explore the key factors impacting the future of the local markets for flexibility and energy, develop plausible future scenarios for the local markets, and analyze their impacts. Thus, the contributions of this paper are: 1) introducing scenario planning methods to provide insight for future developments of emerging concepts in the energy system's area, 2) exploring and ranking the key factors that affect the future of the local markets in Europe (with a focus on Sweden), 3) developing qualitative plausible future scenarios for the local markets, analyzing the impacts of the scenarios, and providing suggestions to address these impacts.

In the rest of this paper, the utilized methodology for scenario planning is elaborated in Section [I] The results including the identified key factors and the developed scenarios are presented in Section IIII. The key impacts from the scenario analysis are discussed and suggestions are provided in Section [V] In Section V, the study is concluded.

\section{Methodology}

The three main schools of techniques for developing scenarios are intuitive logics, probabilistic modified trends methodology, and the French approach La prospective [17], [18]. Each of these techniques has been evolved in different institutes to achieve specific purposes. The intuitive logics school is one of the most dominating methods for scenario development, and has received a lot of attention in the literature for scenario planning [17]. This approach was originally used by Pierre Wack at Shell in the 1960s [17]. The purpose of this method is to make sense of situations and developing strategies, while it can also be an ongoing learning activity [17]. Such purposes match the objective of this study as it is a process-oriented methodology and it aims to provide insights into an on-going learning activity. This approach does not require complex computer-based analysis [17] and can be used as initial input for designing a concept. The output is a set of plausible qualitative scenarios in a narrative form. This set of equally plausible scenarios include strategic options, implications and early warning signals [17] which can be used as input to different stakeholders involved in designing the local markets.

Scenario development approaches for the intuitive logic method are varied in the literature, ranging from five to fifteen steps or more depending on what features of the scenarios are included [17], [18]. The approach used in this study is a seven-step process proposed by Conway [19]. This approach is a more generic form of approaches proposed by Schwartz [20] and the Stanford Research Institute International (SRI) [21], [22]. These approaches are among the most popular and frequently used approaches in intuitive logic school [13], [18]. The seven-step process is as follows:

1) Identify the focal question

2) Environmental scanning- internal and external

3) Selecting drivers of change and ranking them

4) Building the scenario matrix

5) Developing the scenarios

6) Presenting the scenarios

7) Considering the strategic implications

In step one, the focal question needs to be identified. These questions in this study are: 1) what the plausible scenarios for the future of the local energy and flexibility market are, and 2) what the impact of these scenarios would be? In step two, the environment scanning was done by literature review [7], [23], [24], experiences from relevant projects [25], [26], and inputs from four DSOs in Sweden, Switzerland, Turkey, Bulgaria.

In step three, the key driving factors and trends were identified based on the inputs in step two and iterated between a group of experts to finalize the core key factors. The ranking of these key factors was done by two means. A survey was designed to rank the impact and uncertainty of the factors, and a cross-impact matrix was designed to identify the dynamics between these factors and finding the most impactful factors. In the survey, the participants were asked the following questions: Considering the horizon up to the year 2035,1$)$ "what level of uncertainty do you foresee regarding each of the factors and trends?", and 2) "how impactful are these factors and trends in your country on the future of local energy/flexibility trading?". The level of impact and uncertainty is rated based on a Likert scale (from zero to five). Zero represents low impact/uncertainty and five represents high impact/uncertainty. The survey was distributed among experts that have a background and understanding of the concept of local energy and flexibility trade. For filling the cross-impact matrix, the experts were asked to rate the impact of each key factor/trend on other factors (Figure 1). Each cell of the matrix indicates how much the corresponding factor of the row impacts the column's factor. The active score of a factor is the sum of the row corresponding to that factor. A higher active score implies that the factor/trend has a higher cross-impact on the other factors/trends, and therefore it can be relatively more important. The cross-impact matrix was filled by a smaller group of experts compared to the survey participant because it is more time-consuming for the participants and therefore it received fewer responses.

\begin{tabular}{|c|c|c|c|c||c|}
\hline F & F1 & F2 & F3 & Active score & 0: Indipendent \\
\hline F1 & & 1,0 & 1,0 & 2,0 & 1: Slight impact \\
\hline F2 & 0,0 & & 1,0 & 1,0 & 2: Dependent \\
\hline F3 & 2,0 & 3,0 & & 5,0 & 3: Strong driver \\
\hline
\end{tabular}

Fig. 1. A cross-impact matrix and the active score.

In step four, a four-quadrant scenario matrix was built based 
on the received inputs in step three. To build the scenario matrix (Figure 2p), the two most uncertain and impactful factors must be chosen. This was done by ranking the survey results (Figure 2a) and further narrowed down by considering the obtained scores from the cross-impact analysis [27]. The two most impactful and uncertain factors in Figure 2 were used for forming the two axes of the scenario matrix in Figure 2 p. The most impactful but less uncertain factors are highly suggested to be considered while designing the project outputs. Factors with high uncertainty but low impact are secondary issues. Less impactful and less uncertain factors are to monitor and reassess in case of need.

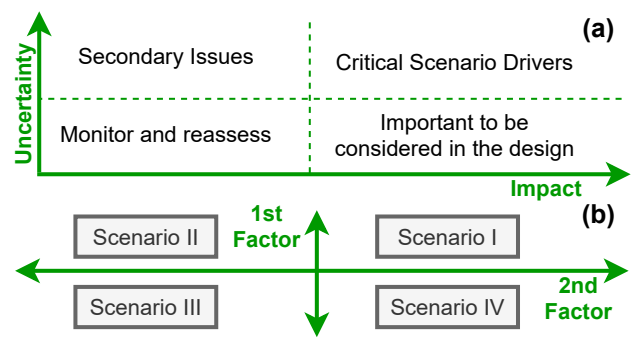

Fig. 2. Factors ranking and scenario matrix: (a) uncertainty-impact ranking (modified figure from [13], [19]), (b) scenario matrix based on the two most uncertain and impactful factors

In step five, the four different worlds (scenarios) from step four were further assessed and described to build a narrative and check if they make sense to the group. In step six, the results were formed in a presentable format. Lastly, in step seven, the implications of the scenarios, including their impact and characteristics were identified and described accordingly by means of workshops and iterations among the group of experts involved in the task.

\section{SCENARIOS}

The final trends and key factors for the future of the concept that were selected based on the methodology (steps 2 and 3). The uncertainty and impact of these factors were evaluated by surveying 14 experts from different stakeholders in different EU countries (the majority from Sweden). The cross-impact of the same factors were analyzed internally in a smaller group of experts on the topic. The key factors and the results from the survey and cross-impact analysis can be seen in Table I.

To be able to develop the scenarios, we need to rank the factors to find the most uncertain and impactful elements. For ranking, the results from the survey are grouped into four groups based on Figure 2 a. The result is presented in Figure 3 where the two dashed lines that group the factors are the average of uncertainty or impact scores. The critical scenario drivers (in the up-right corner) are factor numbers $8,11,15,9$, and 3 . The two axes of the scenario matrix (Figure 2p) need to be selected from these five critical factors. The active scores from the cross-impact analysis were used to filter further the critical scenario drivers. Among the critical scenario drivers (factor numbers $8,11,15,9$, and 3), factor 11,8 , and 15 have the highest active scores of $32.7,30$, and 29.7. Since the active score of factors 8 and 15 are very close and factors 11 and 8 are directly related to end-users, one axis was chosen to include critical factors related to end-users (i.e. factor numbers 11 and 8). The second axis was chosen to be based on factor number 15 which is related to the regulatory incentives for the DSOs. Accordingly, the four plausible future scenarios for the local markets were developed (Figure 4 .

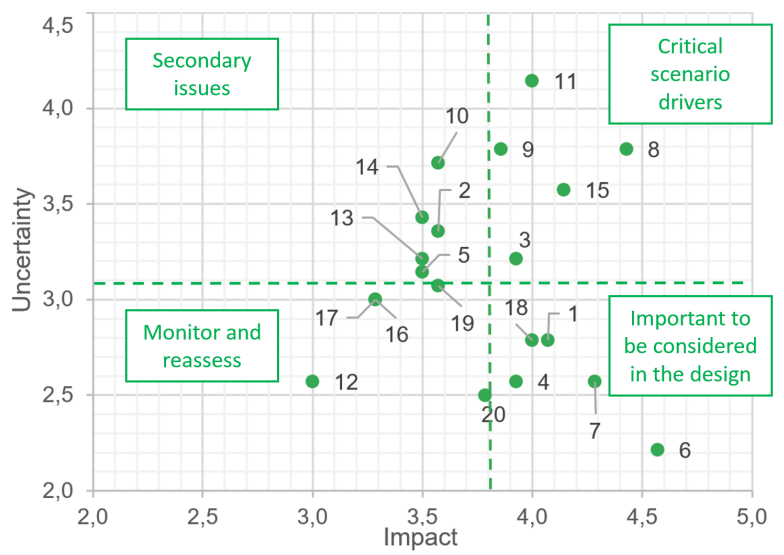

Fig. 3. Results for uncertainty-impact ranking: each point is a factor/trend in Table I] and the separating lines are the average of all uncertainty or impact scores for all factors/trends.

In Figure 4 the Y-axis represents two characteristics of endusers. The first characteristic is whether end-users are willing to participate in local markets (being active or passive), and the second one is if end-users are automated, digital, and can have a fast and precise control over their energy assets or not (being smart or conventional). The $\mathrm{X}$-axis represents the existence of regulatory incentives for DSOs to promote the local markets. Due to the monopoly nature of DSOs, there are regulations that financially regulate DSOs. These regulations can favor capital expenditures over operational costs. Therefore, investing in the infrastructure can be financially more attractive to DSOs rather than using operational measures such as local markets. Moreover, developing local markets can be more challenging for DSOs compared to traditional investments which might not be interesting if not financially beneficial [23]. So, changes in the regulatory framework can incentivize DSOs to deploy local markets or not. Based on these axes, four plausible future scenarios for the concept are formed and explained below.

\section{A. Scenario I}

Scenario I is the most suitable future for the local energy and flexibility markets among the developed four scenarios. In this future, the end-users are digitalized and smart. They have automated, fast, and precise control over their energy assets such as distributed generation (DG), batteries, heat pumps, and controllable loads, and they are willing to actively participate in the local markets. Furthermore, the cost-effectiveness and functionality of the local markets are communicated adequately with regulators and the involved stakeholders. As a result of this communication, regulatory incentives are in place for DSOs to participate and promote these local markets. 
TABLE I

ANALYZED KEY FACTORS/TRENDS AND THEIR UNCERTAINTY (U), IMPACT (I), AND ACTIVE (A) SCORE. A HIGHER ACTIVE SCORE SHOWS MORE IMPACT FROM THE FACTOR ON OTHER FACTORS.

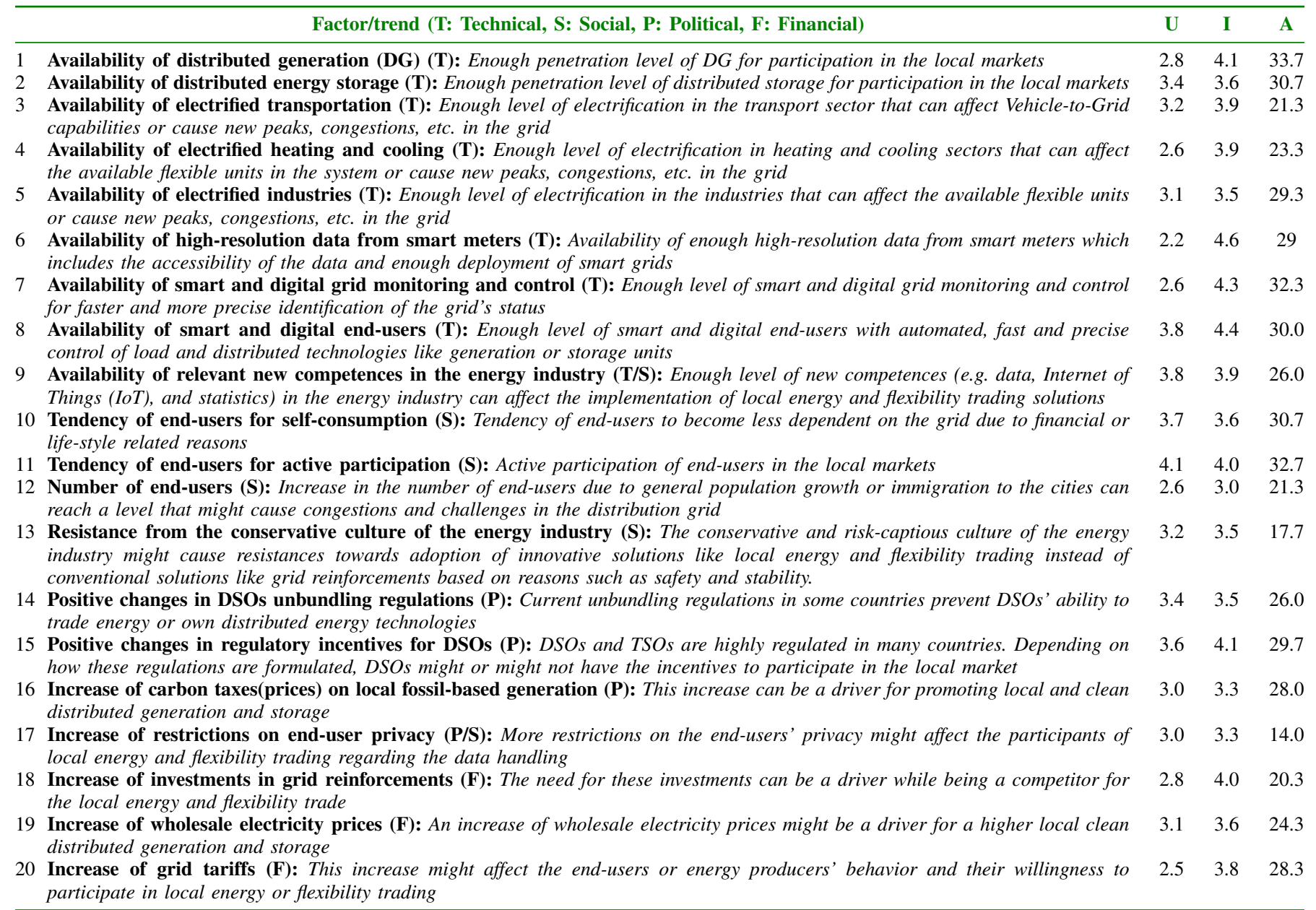

Due to the willingness and smartness of the end-users, recruiting participants is relatively easier. In this scenario, the number of participants can be larger. Thus, the concerns about market liquidity or market power exercises are less. This scenario can have good potentials for implementing peer-topeer trading structures. On the other hand, because of the high number of participants which can be private individuals, a well-designed process is required for the qualification of the participants to avoid issues such as the inability to deliver the cleared flexibility or energy. This qualification process can be costly and complicated for market operators and participants. Moreover, because of the high number of small individual participants, the scalability and computational burdens from the high number of participants need to be examined carefully.

\section{B. Scenario II}

The previous characteristics for the smartness and activeness of the end-users are valid for scenario II. However, there are no regulatory incentives for the DSOs to participate or promote the local markets. Due to the activeness and smartness of endusers, this scenario has a good potential for implementation of peer-to-peer structures while the concerns regarding scalability, computational burden, and qualification procedures exist.
To have a more implementable market design in such a future, it is important to consider other possible consumers of flexibility/energy (e.g. balancing responsible parties, or TSOs) while designing the solution. Besides, proactive communication and share of experience with DSOs play an important role in reforming this scenario towards Scenario I.

In this scenario, dynamic tariffs and other new tariff designs are the competitors to the concept of the local market. This is because the end-users are smart and active and capable of reacting to such tariff designs and local markets do not have any regulatory superiority over the new tariff designs.

\section{Scenario III}

This scenario is a challenging form of future for the concept of local markets and similar to where we are today. The end-users are considered as passive players that are not willing to participate in the local trade. Moreover, the level of digitalization, smartness and control is limited in the end-users. These characteristics of the end-users can also be translated to special regions or archetypes in a city, or the initial phase for implementation of local markets. Similar to scenario II, it does not benefit from regulatory incentives for DSOs. 


- Acenario II: Pilot projects/intermediary adoption phases
$\begin{aligned} & \text { - Higher plausibility for peer-to-peer solutions } \\ & \text { - Higher costs for qualification processes }\end{aligned}$

Fig. 4. Scenario matrix for future of local energy and flexibility trade

For a successful implementation of the concept in this scenario, end-users need to be digitalized and the ease-ofuse needs to be considered for recruiting participants. The intermediary actors (e.g. aggregators) that facilitate the participation of end-users play an important role in this scenario. An example of providing smartness while recruiting end-users is Voltalis [28]. Voltalis provides end-users a box that controls the heating in the apartment. The end-users can opt-out of participating by just pressing a button. The box, its installation and maintenance are free-of-charge. Such extra costs for aggregators might result in higher prices for flexibility/energy, which highlights the importance of developing cost-effective business plans for these actors. Moreover, the limited number of participants/aggregators might cause other challenges such as market illiquidity and market power practices.

In this scenario, other solutions like active grid control methods or grid reinforcements are among the competitors for the concept of local markets. Moreover, peer-to-peer solutions might face difficulties in implementation due to the unwillingness or unavailability of smart end-users for participation.

\section{Scenario IV}

The last scenario is a possible future realization where the DSOs are incentivized by regulations while end-users have remained passive and conventional. In this scenario, the DSOs can play an active role besides aggregators in digitalizing and recruiting the end-users. Like the previous scenario, the endusers are not willing to participate actively in the market; therefore, the main supplier of the flexibility/energy would be aggregators or large industrial end-users.

As the DSOs are more willing to promote local markets, instruments such as capacity markets or long-term contracts can be designed with the help of DSOs to incentivize investments in the smartness and digitalization of end-users while affecting their willingness to participate in these markets.

\section{KeY IMPACTS AND SUGGESTIONS}

The results from the scenario development for the future of the local energy and flexibility trading have shown that the highest expected impact and uncertainty are regarding the endusers' willingness for participation, their level of digitalization and automation (smartness), and regulatory incentives for adoption of the local markets. Moreover, the results of the survey show that an increase in grid-reinforcement, and the availability of DGs, electrified heating and cooling, smart and digitalized grid monitoring and control tools, and highresolution data have lower uncertainty but high impact on the future of these markets. The results from this work can be used by different stakeholders in the field as an insight into the possible key factors. However, they are not necessarily a forecast of the future for these markets.

Considering the above-mentioned key results, DGs, EVs, and electrified heating and cooling would possibly be available to a certain extent on the end-user side. However, the key concern is whether these assets are accessible to be involved in the local markets or not. The key concern is arising from, first, the willingness of the end-users for participation and, second, the end-users' level of digitalization and automated control for these assets. Therefore, considering different instruments for increasing the level of willingness and smartness of the end-users can be crucial for a successful implementation of these markets. Among others, the possibility of utilizing incentivizing instruments such as capacity markets or longterm contracts, regulations to increase the smartness (e.g. grid codes), or investments by aggregators or DSOs for providing smart switches and IoT platforms to the end-users can be considered. The incentivizing instruments can be built between DSOs and end-users, DSOs and aggregators, or aggregators and end-users. In addition, avoiding complex market structures, developing modular and API-based designs for different system components and algorithms, developing user-friendly and automated designs for control systems at the end-user level are suggested to further facilitate market participation.

Regulatory incentives to promote local markets have shown a great impact on the future of local markets. The existence of these incentives by the year 2030 is relatively uncertain. Therefore, proactive communication with system operators and regulators are suggested to share the experience and the results. This communication can help smoothening the adoption phase and leads to a faster transition from the worst scenario (scenario III) to the preferable scenario (scenario I). 
In the survey results, the impact of distributed energy storage technologies is rated low with a high uncertainty for a considerable penetration level. Due to the intermittent essence of renewable DG, and also a considerably high active score of distributed storage in the cross-impact analysis, it is important to further evaluate such technologies to obtain a better insight into the impact and uncertain role of them in local markets.

Regarding the scenario I and II, better market liquidity can be obtained with a higher number of participants. However, the computational burden and higher qualification processes' costs can cause scalability issues. Therefore, considering these aspects are suggested when designing the system.

Finally, other solutions such as innovative tariff designs, direct grid control, and grid reinforcements can be used alongside or instead of local markets. To maximize the value for all stakeholders and society, considering these other solutions is recommended for building comprehensive approaches to resolve the expected challenges in the most efficient manner.

\section{Conclusions}

In this paper, a well-established method in intuitive logics school of scenario planning is presented and applied for local energy and flexibility markets. The key factors that impact the future of the local markets in Europe (with a focus on Sweden) are explored and ranked using this method. Four plausible future scenarios were developed for the local markets based on these factors, and the key impacts from these scenarios were discussed and suggestions were provided accordingly.

The results show that the availability of active and smart end-users, and possible regulatory incentives for promoting the local markets play important roles in the future of local markets but yet comparatively with a higher uncertainty in their existence. The activeness of the end-users reflects the willingness of them to participate in the local markets and the smartness of end-users reflects their technical capabilities to participate in such systems. The technical possibility for participation can include automation, digitalization, and having a fast and precise control over different distributed energy assets. Other impactful factors in the future of local markets that have less uncertainty in their existence are the availability of high-resolution data, the availability of smart and digital grid monitoring and control, the availability of distributed generation, an increase of investments in grid reinforcements, and the availability of electrified heating and cooling.

\section{ACKNOWLEDGMENT}

The authors want to acknowledge all the FLEXIGRID project partners who participated in the survey and interviews.

\section{REFERENCES}

[1] J. Villar, R. Bessa, and M. Matos, "Flexibility products and markets: Literature review," Electric Power Systems Research, vol. 154, pp. 329340, 2018.

[2] X. Jin, Q. Wu, and H. Jia, "Local flexibility markets: Literature review on concepts, models and clearing methods," Applied Energy, vol. 261, p. $114387,2020$.

[3] D. S. Kirschen and G. Strbac, Fundamentals of power system economics. John Wiley \& Sons, 2018.
[4] A. Delnooz, J. Vanschoenwinkel, E. Rivero, C. Madina, and P. Junco, "D1. 3-definition of scenarios and products for the demonstration campaigns," Coordinet project, Tech. Rep., 2019.

[5] P. Olivella-Rosell, E. Bullich-Massagué, M. Aragüés-Peñalba, A. Sumper, S. Ø. Ottesen, J.-A. Vidal-Clos, and R. VillafáfilaRobles, "Optimization problem for meeting distribution system operator requests in local flexibility markets with distributed energy resources," Applied energy, vol. 210, pp. 881-895, 2018.

[6] N. Mirzaei Alavijeh, D. Steen, Z. Norwood, A. Le Tuan, C. Agathokleous et al., "Cost-effectiveness of carbon emission abatement strategies for a local multi-energy system - a case study of chalmers university of technology campus," Energies, vol. 13, no. 7, p. 1626, 2020.

[7] P. Olivella-Rosell, P. Lloret-Gallego, Í. Munné-Collado, R. VillafafilaRobles, A. Sumper, S. Ø. Ottessen, J. Rajasekharan, and B. A. Bremdal, "Local flexibility market design for aggregators providing multiple flexibility services at distribution network level," Energies, vol. 11, no. 4 p. $822,2018$.

[8] S. Beucker, H. Doderer, A. Funke, C. Koch, H. Kondziella, J. Hartung, S. Maeding, H. Medert, G. Meyer-Braune, M. Rath, and N. Rogler, "Flexibility, markets and regulation: Insights from the WINDNODE reality lab," WindNODE project coordination, Tech. Rep., Jan. 2021.

[9] G. Ringland, Scenarios in business. Wiley, 2002.

[10] S. P. Schnaars, "How to develop and use scenarios," Long range planning, vol. 20, no. 1, pp. 105-114, 1987.

[11] T. J. Stewart, "Dealing with uncertainties in mcda," in Multiple criteria decision analysis: state of the art surveys. Springer, 2005, pp. 445-466.

[12] K. Chen, Z. Ren, S. Mu, T. Q. Sun, and R. Mu, "Integrating the delphi survey into scenario planning for china's renewable energy development strategy towards 2030," Technological Forecasting and Social Change, vol. 158, p. 120157, 2020.

[13] S. Kuiper, "The future of the dso enexis: a research on the strategic position of the dso in 2030 using scenario and trend analysis," Master's thesis, University of Twente, 2015.

[14] R. Alizadeh, P. D. Lund, A. Beynaghi, M. Abolghasemi, and R. Maknoon, "An integrated scenario-based robust planning approach for foresight and strategic management with application to energy industry," Technological Forecasting and Social Change, vol. 104, pp. 162-171, 2016.

[15] S. W. Schuckmann, T. Gnatzy, I.-L. Darkow, and A. Heiko, "Analysis of factors influencing the development of transport infrastructure until the year 2030 - a delphi based scenario study," Technological Forecasting and Social Change, vol. 79, no. 8, pp. 1373-1387, 2012.

[16] Y. Shiftan, S. Kaplan, and S. Hakkert, "Scenario building as a tool for planning a sustainable transportation system," Transportation Research Part D: Transport and Environment, vol. 8, no. 5, pp. 323-342, 2003.

[17] R. Bradfield, G. Wright, G. Burt, G. Cairns, and K. Van Der Heijden, "The origins and evolution of scenario techniques in long range business planning," Futures, vol. 37, no. 8, pp. 795-812, 2005.

[18] M. Amer, T. U. Daim, and A. Jetter, "A review of scenario planning," Futures, vol. 46, pp. 23-40, 2013.

[19] M. Conway, "An introduction to scenario planning," in Foresight Methodologies Workshop, 2003, p. 99.

[20] P. Schwartz, The art of the long view: paths to strategic insight for yourself and your company. Currency, 1996.

[21] W. R. Huss and E. J. Honton, "Scenario planning-what style should you use?" Long range planning, vol. 20, no. 4, pp. 21-29, 1987.

[22] J. Ogilvy and T. Mandel, "How to construct and use scenarios," 1986.

[23] H. Pihl, J. Rossi, and M. Edvall, "D2.1, Report on barriers for adoption of innovative market design,” FLEXIGRID project, Tech. Rep., 2020.

[24] CEER, "Guidelines of good practice for flexibility use at distribution level,” Tech. Rep., 2017.

[25] A. Wolf, C. Sandels, and M. Shepero, "Lokal energilagring eller traditionella nätförstärkningar," Power Circle, Tech. Rep., 2018.

[26] N. Etherden, Y. Ruwaida, and S. Johansson, "CoordiNet D4.5 - Report on lessons learned, bug fixes and adjustments in products and routines within the Swedish demo," CoordiNet project, Tech. Rep., 2020.

[27] S. A. van't Klooster and M. B. van Asselt, "Practising the scenario-axes technique," Futures, vol. 38, no. 1, pp. 15-30, 2006.

[28] Voltalis. Voltalis, first operator of Internet of Energy. [Online]. Available: http://www.voltalis.fr/individual\#header 